\title{
Arrhenius-type temperature dependence of the segmental relaxation below $T_{g}$
}

\author{
Paul A. O'Connell a) and Gregory B. McKenna ${ }^{\text {b) }}$ \\ Polymers Division, National Institute of Standards and Technology, Gaithersburg, Maryland 20899
}

(Received 10 December 1998; accepted 12 March 1999)

\begin{abstract}
In a recent paper DiMarzio and Yang [J. Res. Natl. Inst. Stand. Technol. 102, 135 (1997)] predicted that transport properties such as viscosity and diffusion coefficient do not follow the typical Williams, Landel, and Ferry (WLF) [J. Am. Chem. Soc. 77, 3701 (1955)] or Vogel-Fulcher-type of temperature dependence as the glass transition is approached. Rather, a transition to an Arrhenius-type of temperature dependence is predicted. Here we describe long term aging experiments that explore the temperature dependence of the viscoelastic response of polycarbonate in the vicinity of the glass transition. Aging the material for long times below the nominal glass transition temperature, assures that equilibrium is attained and we can directly test the DiMarzioYang prediction. In tests in which glassy samples of polycarbonate were aged into equilibrium at temperatures up to $17^{\circ} \mathrm{C}$ below the conventionally measured glass transition temperature, we find that the results are consistent with a transition from Vogel-Fulcher or WLF-type behavior to Arrhenius-type behavior. Our results are discussed within the context of other measurements on nonpolymeric glasses and other recent results on polymeric glass formers.
\end{abstract}

[S0021-9606(99)50222-5]

\section{INTRODUCTION}

In the vicinity of the glass transition temperature $T_{g}$ the viscosity and diffusion coefficient of polymers and other glass forming liquids are strong functions of temperature. It is generally accepted that most materials exhibit nonArrhenius behavior. ${ }^{1-5}$ For example, the viscosity $\eta$ is often described using the so-called Vogel-Fulcher ${ }^{3}$ temperature law,

$$
\eta=\eta_{0} e^{B /\left(T-T_{\infty}\right)},
$$

where $\eta_{0}$ is a prefactor, $B$ is a material parameter, $T$ is absolute temperature, and $T_{\infty}$ is the temperature at which the viscosity would become infinite. A typical example of the Vogel-Fulcher-type temperature dependence for polystyrene $^{6}$ is shown in Fig. 1 . The viscosity singularity at $T_{\infty}$ is generally found to be approximately $50{ }^{\circ} \mathrm{C}$ below the conventionally measured glass transition temperature and we note that when $T_{\infty}=0 \mathrm{~K}$, the Vogel-Fulcher expression is equivalent to an Arrhenius equation. The singularity in the viscosity is often interpreted in terms of free volume $e^{1,4,5,7}$ models or the Adam-Gibbs ${ }^{8}$ relation between viscosity and configurational entropy. However, in a recent publication DiMarzio and Yang $^{9}$ presented a new theoretical result, based on configurational entropy concepts, which suggests that there is no singularity in the viscosity-temperature behavior in going through the glass transition. Rather, they predict that the material should exhibit a transition towards an Arrhenius-type temperature dependence as the $T_{g}$ is ap-

\footnotetext{
a)Current address: IRC for Polymer Physics, University of Leeds, Leeds, United Kingdom.

b) Author to whom correspondence should be addressed. Electronic mail: gmckenna@nist.gov
}

proached. Therefore, it is of interest to revisit the problem of the viscosity-temperature relationship near to and below the nominal glass transition temperature.

In examining the problem of the temperature dependence of the transport properties (e.g., viscosity, diffusion coefficient) in glass forming systems we need to consider several aspects of the behavior. First, in most nonpolymeric materials, the viscosity and the material "glassy" relaxation times will follow closely with each other. On the other hand, in polymers, the viscosity is dominated by entanglement ${ }^{1}$ effects with the result that the temperature dependence of the glassy or segmental relaxations may be different from the terminal relaxations or viscosity, ${ }^{10-14}$ i.e., both $B$ and $T_{\infty}$ in Eq. (1) can differ depending on whether one measures segmental or terminal relaxation times. To assure that we address the relaxations relevant to the glass, we use timetemperature superposition principles ${ }^{1}$ to determine shift factors for the segmental relaxation of polycarbonate. We then ask if these shift factors follow the appropriate VogelFulcher-type behavior or take on an Arrhenius-type behavior as one goes below the conventional glass transition.

An additional consideration is that, as one traverses the glass transition, it is well known that the material falls out of equilibrium and the corresponding relaxation times or viscosities of the nonequilibrium system do not become singular. ${ }^{5,15-22}$ Therefore, it is important that measurements of the relaxation response be performed in such a way that the equilibrium response is obtained. We assure this by performing physical aging experiments using Struik's ${ }^{22}$ protocol (described subsequently) for times as long as $23 d(2$ $\times 10^{6} \mathrm{~s}$ ) to assure equilibration of the mechanical response.

Finally, we note that there are data in the literature that suggest Arrhenius behavior for the viscosity ${ }^{23,24}$ in some 


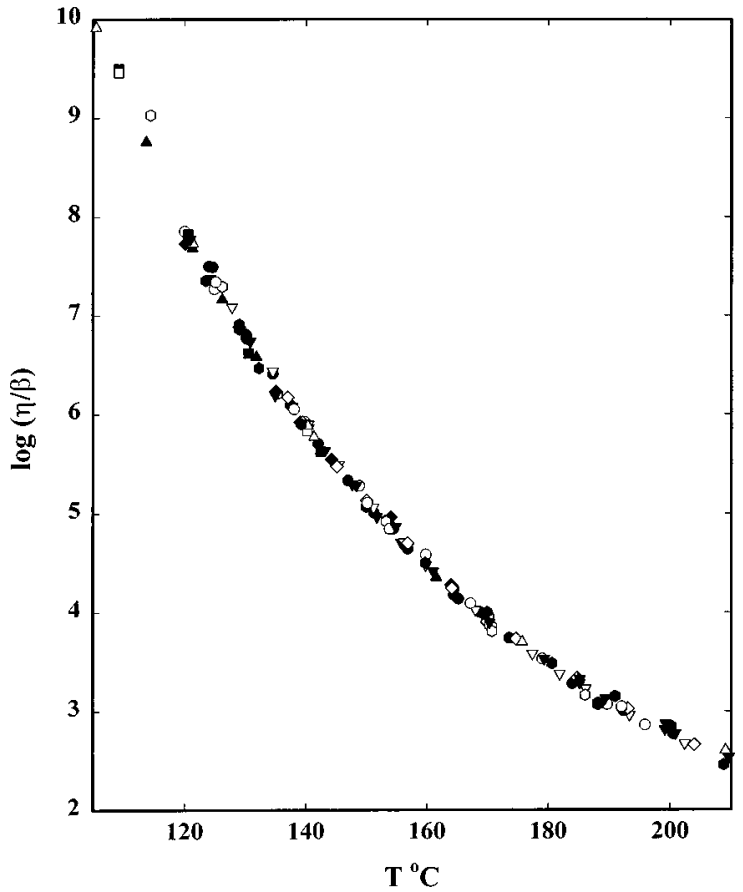

FIG. 1. Typical viscosity-temperature dependence for a polymeric melt. See text for discussion (after Ref. 6).

small molecule glass forming liquids and for the structural recovery times ${ }^{25}$ in polymeric glass formers. We discuss our results in terms of these findings as well.

\section{METHODS OF ANALYSIS}

\section{A. Analysis of the stress relaxation data}

A standard approach to characterize the viscoelastic response of a polymer glass, such as polycarbonate, is to perform stress relaxation measurements in torsion. Here we report the relaxation response at a nominal torsional strain of 0.02 , which we found previously ${ }^{18}$ to exhibit the same aging time and temperature behavior as experiments at smaller strains, although at this strain the polycarbonate may be slightly into the nonlinear response regime. An often-used representation for the response function in stress relaxation experiments is the stretched exponential of Kohlrausch $^{26}$-Williams-Watts ${ }^{27}$ (KWW),

$$
G(t)=G_{0} e^{-\left(t / \tau_{0}\right)^{\beta}},
$$

where $G(t)$ is the shear (torsional) modulus response at time $t, \tau_{0}$ is a characteristic time, $\beta$ a shape parameter related to the breadth of the relaxation curve, and $G_{0}$ can be interpreted as the zero time shear modulus. Clearly, $G(t)$ is a function of both aging time in the glassy state and of temperature. The prior study ${ }^{18}$ showed that time-aging time superposition and time-temperature superposition applied very well to this polycarbonate in the range $30-135^{\circ} \mathrm{C} .{ }^{28}$

\section{B. Time-aging time and time-temperature superposition}

The reduced time concepts involved in the time-aging time and the time-temperature superposition principles have been extensively discussed elsewhere. $1,5,10,12-15,18-22,27,29,30$ Here we provide the relevant definitions for the analysis used in this study. The superposition principle is based on the assumption that the shape of the viscoelastic spectrum, and therefore that of the relaxation modulus, does not vary with the relevant parameter, e.g., aging time $t_{e}$ or temperature $T$. In the framework of the KWW function [Eq. (2)], the isothermal response at aging time $t_{e, i}$ relative to that at some reference aging time $t_{e, r}$ is written in terms of an aging time shift factor $a_{t e}$,

$$
a_{t e}=\frac{\tau_{0}\left(T, t_{e, i}\right)}{\tau_{0}\left(T, t_{e, r}\right)} .
$$

Similarly, the isochronal response at different temperatures is described by a temperature shift factor $a_{T}$,

$$
a_{T}=\frac{\tau_{0}\left(t_{e}, T_{i}\right)}{\tau_{0}\left(t_{e}, T_{r}\right)},
$$

where $T_{i}$ is the temperature of interest and $T_{r}$ is the reference temperature. Then, the simplest form of time-aging time and time-temperature superposition for the stress relaxation response is described as

$$
G\left(t_{e}, T\right)=G_{0} e^{-\left[t / a_{T} a_{t e} \tau_{0}\left(t_{e, r}, T_{r}\right)\right]^{\beta}} .
$$

Equation (5) represents a time-aging time and timetemperature reduction without any "vertical" shifting of the data. However, it has been observed ${ }^{1,12,29}$ that, in fact, vertical shifts can be required to reduce the data and the phenomenological justification for such shifting has been described by Markovitz. ${ }^{30}$ Above the glass transition, in polymers, the basis for the vertical shifts is understood in terms of rubber elasticity of the entangled macromolecular chains. ${ }^{1}$ Near to and below the glass transition, the theoretical understanding for such vertical shifts has yet to be provided. Regardless, the vertical shifts for aging time $b_{t e}$ and temperature $b_{T}$ are written in terms of the parameters of Eq. (2) as follows:

$$
\begin{aligned}
& b_{t_{e}}=\frac{G_{0}\left(t_{e, r}\right)}{G_{0}\left(t_{e}\right)}, \\
& b_{T}=\frac{G_{0}\left(T_{r}\right)}{G_{0}(T)},
\end{aligned}
$$

and the reduced stress relaxation modulus is then written as

$$
G\left(t_{e}, T\right)=b_{T} b_{t_{e}} G_{0}\left(t_{e_{r}}, T_{r}\right) e^{-\left[t / a_{T} a_{t e} \tau_{0}\left(t_{e, r}, T_{r}\right)\right]^{\beta}} .
$$

Equations (2)-(8) provide a useful framework for data analysis. Because the viscosity is not the appropriate variable to use to examine the temperature dependence of the segmental relaxation in high molecular weight polymers, we use the segmental shift factor $a_{T}$ which is more readily analyzed in the form of the so-called WLF (Ref. 4) equation rather than the Vogel-Fulcher ${ }^{3}$ Eq. (1). Thus,

$$
\log a_{T}=\frac{C_{1}\left(T-T_{s}\right)}{C_{2}+\left(T-T_{s}\right)},
$$

where $C_{1}$ and $C_{2}$ are material parameters and $T_{s}$ is a reference temperature, often taken as the glass transition tempera- 
ture. The Vogel-Fulcher parameters and the WLF parameters are related by the following expressions:

$$
\begin{aligned}
& C_{1}=B\left(T_{s}-T_{\infty}\right), \\
& C_{2}=T_{s}-T_{\infty} .
\end{aligned}
$$

Finally, in a recent paper, DiMarzio and Yang ${ }^{9}$ proposed a new model that relates the relaxation response (transport properties) of glass forming polymers based on a simplified energy landscape and in the context of the Gibbs-DiMarzio $^{31,32}$ configurational entropy theory of the glass transition. In their model, contrary to the expectation that one would find a Vogel-Fulcher relationship similar to that obtained via the Adam-Gibbs ${ }^{8}$ construction, they find that the shift factors (or viscosities) follow an Arrhenius-type of behavior as the glass transition is approached. Then, the shift factors should depend on temperature according to the following relationship:

$$
\log a_{T}=-\frac{A F_{c}}{k_{b}}\left(\frac{1}{T}-\frac{1}{T_{g}}\right),
$$

where $A$ is a constant, $k_{b}$ is the Boltzmann constant, and $F_{c}$ is the configurational free energy, which DiMarzio and Yang ${ }^{9}$ find to be constant below the glass transition temperature.

Equation (12) predicts that the transport properties are expected to be nonsingular (and Arrhenius-type) as one traverses the glass transition-contrary to prior thought based on the WLF or Vogel-Fulcher relationship observed for viscosity and often also interpreted in terms of the Adam-Gibbs ${ }^{8,33,34}$ relationship in which the shift factors depend on the reciprocal of the configurational entropy $S_{c}^{-1}$. The relevant variable is the configurational free energy $F_{c}$, which is expected to be a constant below the glass transition.

\section{EXPERIMENTAL METHODS (REF. 35)}

\section{A. Material}

The material employed in this investigation was a General Electric Lexan LS-2, which is an UV stabilized, commercial grade bisphenol-A polycarbonate. This material has been extensively characterized in this laboratory and the reader is referred to the relevant publications ${ }^{18,20,21,36-38}$ for detailed information. Relevant to this work, the nominal $T_{g}$ was measured as $141.3^{\circ} \mathrm{C}$ using differential scanning calorimetry heating at $10^{\circ} \mathrm{C} / \mathrm{min} .{ }^{36}$ (The $T_{g}$ was taken as the midpoint of the change in the heat capacity.) The characteristics of the material are given in Table I. The polycarbonate was supplied in the form of extruded rods of $25 \mathrm{~mm}$ diam.

\section{B. Stress relaxation testing}

Cylinders of the polycarbonate were first machined to a length of $50 \mathrm{~mm}$ and diameter $12 \mathrm{~mm}$. Subsequently a gauge section of $30 \mathrm{~mm}$ length and from 4 to $7 \mathrm{~mm}$ diam was machined. In order to remove the effects of previous thermal and/or mechanical history, the samples were heated to $145^{\circ} \mathrm{C}$ (approximately $4{ }^{\circ} \mathrm{C}$ above the nominal $T_{g}$ ) for $1 \mathrm{~h}$ prior to testing. Residual birefringence was not observed on looking through crossed polars. Single samples could be
TABLE I. Molecular ${ }^{\mathrm{a}}$ characteristics of polycarbonate used in this study (from Ref. 37)

\begin{tabular}{cccc}
\hline \hline $\begin{array}{c}M_{w} \\
(\mathrm{~g} / \mathrm{mol})\end{array}$ & $\begin{array}{c}M_{n} \\
(\mathrm{~g} / \mathrm{mol})\end{array}$ & $\begin{array}{c}M_{z} \\
(\mathrm{~g} / \mathrm{mol})\end{array}$ & $\mathrm{BPA}^{\mathrm{b}}$ \\
\hline 46579 & 18236 & 74220 & 42.8 \\
\hline \hline
\end{tabular}

${ }^{a}$ Molecular weights are given as weight average, number average, and $z$ average by subscripts $w, n$, and $z$, respectively. According to ISO-31-8, the term "molecular weight" has been replaced by "relative molecular mass" symbol $M_{r}$. Thus, if this nomenclature and notation were to be followed in this publication, one would write $M_{r, w}$ instead of the historically conventional $m_{w}$ for the weight average molecular weight and it would be called the "weight average relative molecular mass." The conventional notation, rather than the ISO notation has been used in this publication.

${ }^{\mathrm{b}} \mathrm{BPA}=\mathrm{Bisphenol-A}$ residual in parts BPA per million parts by mass polycarbonate.

used for multiple tests. Samples were replaced when they failed. Therefore, multiple samples were used to complete the entire body of experiments.

The torsion measurements were carried out on a Rheometrics RMS 7200 load frame, modified in our laboratory with a computer controlled servomotor. The sample and grips were housed within a heater chamber for temperature control. The measured oven stability (based on the range of measurements) was better than $\pm 0.2^{\circ} \mathrm{C}$. The torque relaxations were measured at nominal strains $\gamma$ (based on the cylinder outer radius) of 0.02 ,

$$
\gamma=R \Psi, \quad \Psi=\frac{\Phi}{L},
$$

where $\Phi=$ angle of twist, $R$ is the cylinder radius, $L$ is the length of the gauge section, and $\Psi$ is the angle of twist per unit length.

Aging experiments were performed by first normalizing the samples at $145^{\circ} \mathrm{C}$ for $1 \mathrm{~h}$ and then rapidly cooling them to the aging temperature for testing. We followed the aging procedure first suggested by Struik. ${ }^{22}$ After the temperature change, a sequence of deformations was applied to each sample beginning at an aging time $t_{e, 1}$ and with a loading time $t_{d, 1}$ where the sample was unloaded for a time $t_{u, 1}$. Subsequent deformations were applied at $t_{e, i}$ for durations $t_{d, i}$ such that $t_{d, i} / t_{e, i}=0.10$ and $t_{e, i+1}=2 t_{e, i}$. Here we report results from experiments performed for aging temperatures between $70^{\circ} \mathrm{C}$ and $144{ }^{\circ} \mathrm{C}$ with emphasis on results near to the glass temperature where the aging experiments showed that the mechanical response had attained its equilibrium value in $23 d$ or less. For temperatures higher than $135^{\circ} \mathrm{C}$ no aging was observed because the equilibration times were shorter than the shortest aging time $(\approx 1800 \mathrm{~s})$ at which the mechanical testing was performed.

Finally, throughout this work, rather than using the stress relaxation modulus we use the torque response. This is because the absolute values of the modulus are not well known for the samples tested very close to the glass transition temperature. This occurred because the samples are soft near to the glass transition and this can lead to some slippage in the grips upon applying the step-strain in torsion (the grips are fully described in a paper by McKenna and Kovacs ${ }^{39}$ ). Hence the absolute magnitude of the strain is unknown. 


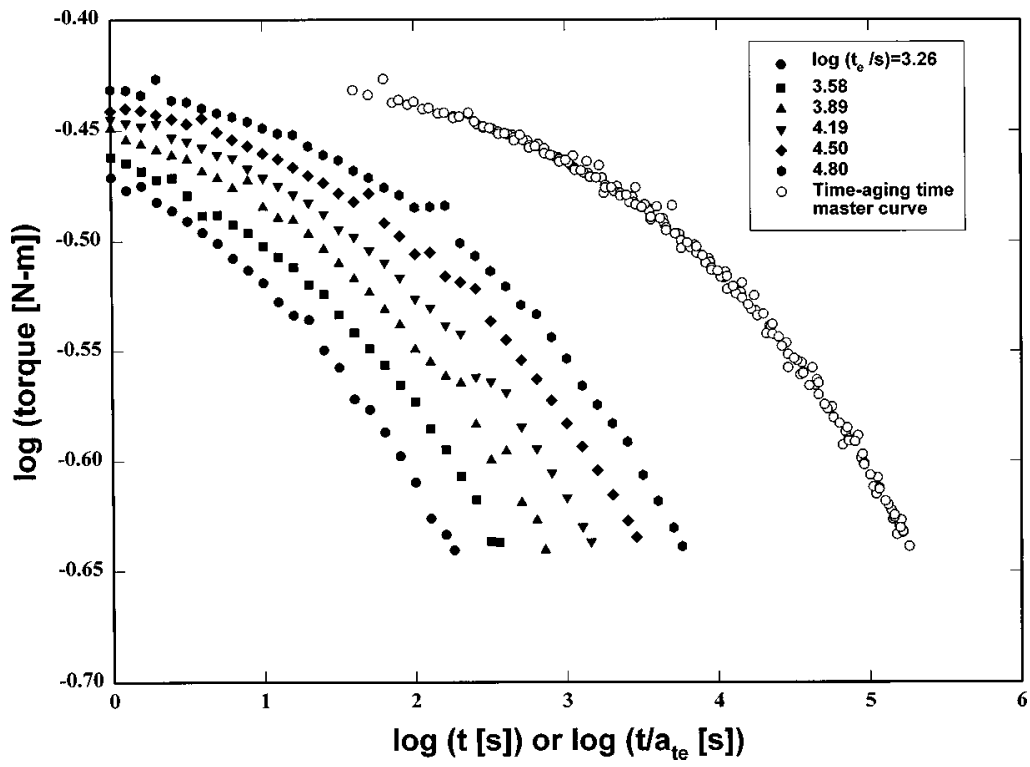

FIG. 2. Torsional relaxation response of polycarbonate at different aging times subsequent to a quench from $145^{\circ} \mathrm{C}$. Aging times as in legend. The master curve is offset by 1.5 decades for clarity. See text for discussion and error estimates.

However, for the purposes of determining the timetemperature shift factors, the shape of the curves is unchanged and the data are valid for these determinations. We add that, if the slippage that occurred during the initial step in strain had continued during the duration of the relaxation, the shapes of the relaxation curves would have been distorted and time-temperature (or time-aging time) superposition could not have been applied to the data and shift factors would not have been able to be obtained. One result of this, however, is the inability to determine the vertical shift factors because these are impacted by the error in the strain magnitude. This procedure is, then, similar to that used by Plazek $^{40}$ in creep measurements in which an empirical geometry factor is applied for samples of unknown geometry and, therefore, for which the strains are unknown. This results in an arbitrary vertical shifting of the data.

\section{RESULTS}

In prior $^{18,37,41}$ work on this polycarbonate material we applied time-aging time and time-temperature superposition to data generated from tests carried out at temperatures between $30^{\circ} \mathrm{C}$ and $135^{\circ} \mathrm{C}$ and for aging times to $63000 \mathrm{~s}$. (The emphasis of the current work is on new data obtained for samples that have been aged into equilibrium below the glass transition temperature.) Figure 2 shows the typical relaxation responses for a sample tested at $70^{\circ} \mathrm{C}$ after being quenched from $145^{\circ} \mathrm{C}$. As seen in the figure, the relaxation modulus shifts along the log (time) axis and the conditions for timeaging time superposition are obtained. Both vertical and horizontal shifts were necessary to superimpose our data. The master curve is depicted as offset by a decade and a half in time for clarity and to show the quality of the superposition. This illustrates the time-aging time superposition behavior of this material.

The aging time shift factors obtained near to the glass transition temperature by shifting torque data are depicted in Fig. 3 where we have plotted the logarithm of the aging time shift factor vs the logarithm of the aging time for temperatures between $119^{\circ} \mathrm{C}$ and $135^{\circ} \mathrm{C}$. Note that the shifts are arbitrarily set to zero at $1800 \mathrm{~s}$ aging time. Several important points arise from Fig. 3. First, at $135^{\circ} \mathrm{C}$ we note that the material has attained its equilibrium at the shortest aging time after the quench (approximately $30 \mathrm{~min}$ ), even though the glass transition is nominally $141^{\circ} \mathrm{C}$. This simply reflects the kinetic nature of the $T_{g}$ and is consistent with other such observations. ${ }^{15,39,42,43,44}$ As the temperature of aging decreases, we see that the shift factors (or relaxation times) evolve as aging time increases because of the decreasing volume or enthalpy of the nonequilibrium glass. ${ }^{5,22}$ After some time the shift factors cease changing and the time at which the evolution ceases increases strongly with decreasing temperature. We consider the plateau for which the evolution has ceased to be the equilibrium mechanical response

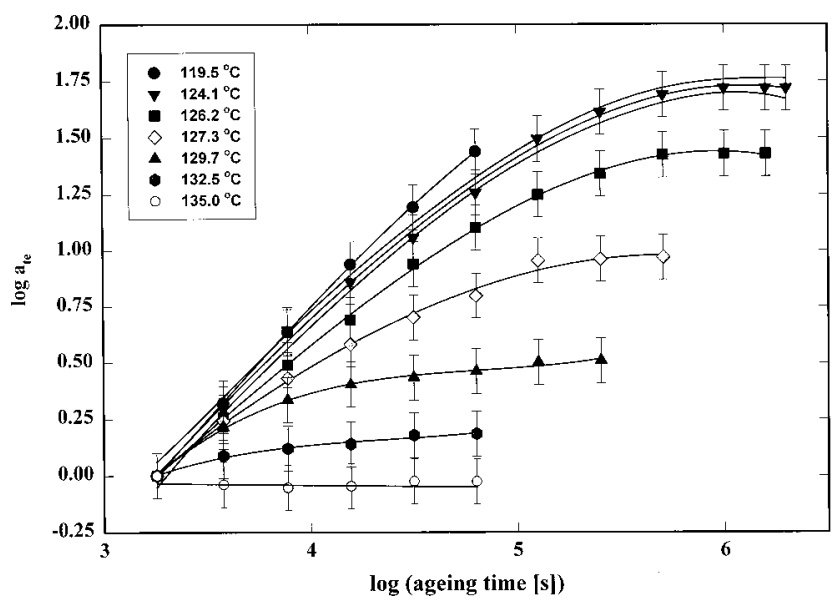

FIG. 3. Variation of the aging time shift factor with aging time for temperatures between $119^{\circ} \mathrm{C}$ and $135^{\circ} \mathrm{C}$. Note that at $135^{\circ} \mathrm{C}$, the sample has equilibrated within the first $1800 \mathrm{~s}$ after the quench and that equilibration takes longer and longer as temperature is decreased. The lowest temperature for which equilibration is achieved was $124.1^{\circ} \mathrm{C}$. The approximate equilibration time exceeded 12 days $\left(1 \times 10^{6} \mathrm{~s}\right)$. Error bars represent \pm 0.1 logarithmic decade and the lines bounding the data for the $124.1{ }^{\circ} \mathrm{C}$ experiments represent $95 \%$ confidence limits for the 3rd order polynomial regression to the data. 


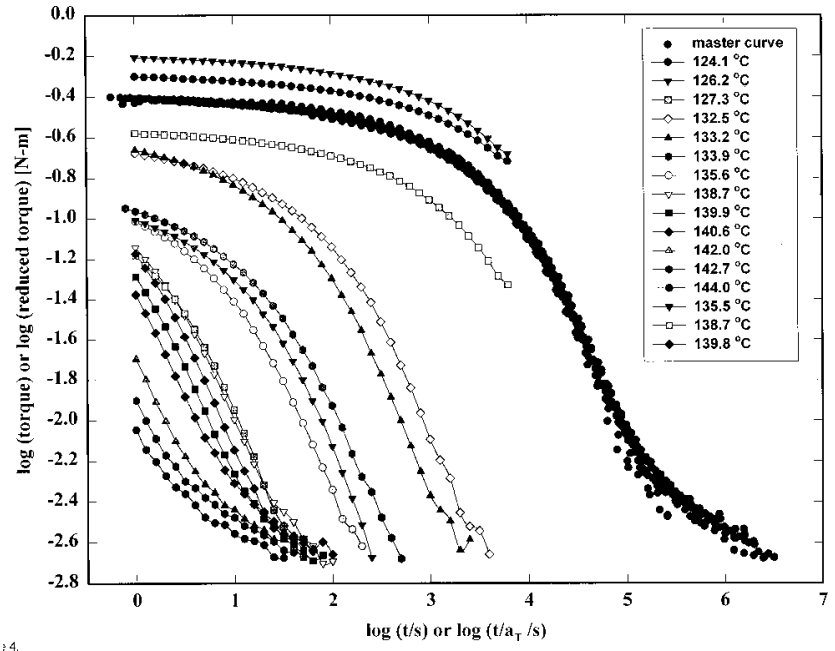

FIG. 4. Torsional relaxation response of polycarbonate for temperatures from $124.1{ }^{\circ} \mathrm{C}$ to $144.0^{\circ} \mathrm{C}$. Tests are all for samples that were aged into equilibrium. Some temperatures are omitted for clarity. Master or reduced curve, which includes data from all temperatures, was constructed using horizontal (time) shift factors presented in Figs. 5 and 6. (See text for error estimates.) In addition, vertical shift factors to correct for the uncertainty in the strain were used. Master curve is offset by 5 decades for clarity and the reference temperature is $144{ }^{\circ} \mathrm{C}$.

and we see that at $124.1^{\circ} \mathrm{C}$ the time to reach this plateau is on the order of 12 days $\left(1 \times 10^{6} \mathrm{~s}\right)$.

In order to compare responses at the different temperatures, time-temperature superposition needs to be valid. As noted above, prior work ${ }^{18,37,41}$ established that this polycarbonate can be described by time-aging time and timetemperature superpositions over the temperature range 30$135^{\circ} \mathrm{C}$. Here we report the new torque results for the samples aged long enough that their mechanical response had ceased to change, i.e., into equilibrium. Figure 4 shows individual temperature curves as well as the reduced or master curve created by performing vertical and horizontal shifts on the data. The temperature shift factors and error estimates for the equilibrated samples are presented subsequently. ${ }^{28}$

Finally, the data obtained from the time-temperature reduction can be used to test the DiMarzio-Yang ${ }^{9}$ expectation that the temperature dependence of the time shift factors should become Arrhenius as the glass transition is traversed. In Fig. 5 we plot the temperature shift factors with $142^{\circ} \mathrm{C}$ chosen as the reference temperature, i.e., $a_{T}=1\left(\log a_{T}=0\right)$. The error in the temperature is approximately $\pm 0.2^{\circ} \mathrm{C}$ (range of measurements) and reflects the stability of the heater chamber. The error in the temperature shift factor is approximately $\pm 0.2 \log$ units. This error is the estimated sample to sample variation determined from the shift factors required to superpose repeat experiments carried out at the same temperature. Also plotted is the extrapolation of the WLF or Vogel-Fulcher equation for the segmental relaxation of polycarbonate given by Mercier and Groenincks ${ }^{45}$ from stress relaxation measurements at temperatures from $140.5^{\circ} \mathrm{C}$ to $172.5^{\circ} \mathrm{C}$ and shifted to $T_{s}=142^{\circ} \mathrm{C}\left(C_{1}=14.42\right.$ and $\left.C_{2}=43.97^{\circ} \mathrm{C}\right)$. The "universal" WLF curve $\left(C_{1}\right.$ $=17.44$ and $C_{2}=51.6^{\circ} \mathrm{C}$ shifted to $T_{s}=142{ }^{\circ} \mathrm{C}$ ) is also shown.

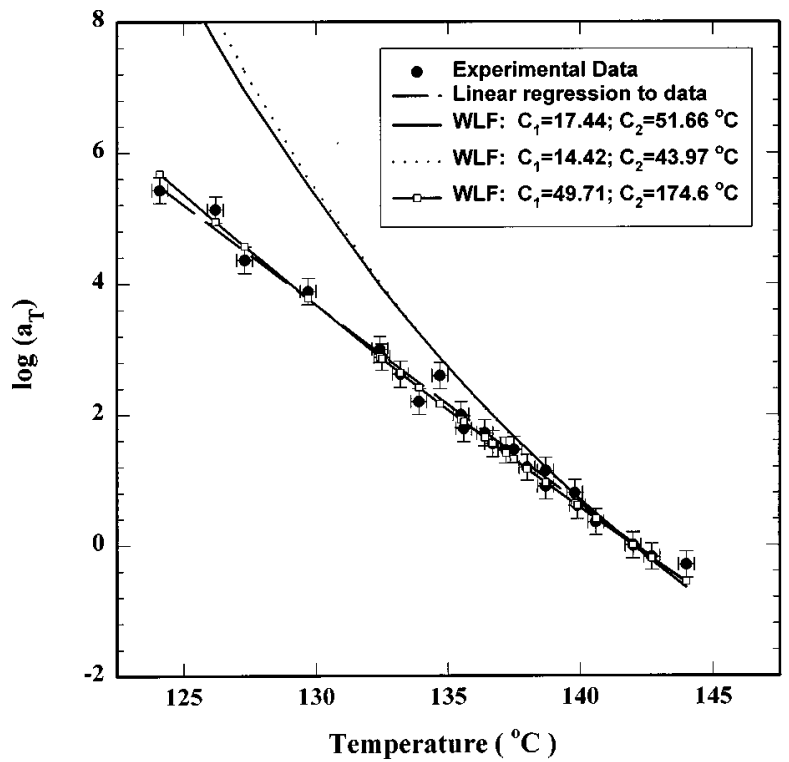

FIG. 5. Comparison of the temperature shift factors obtained for polycarbonate with linear and WLF behaviors, as indicated in legend. Samples aged into equilibrium in the vicinity of the calorimetric glass transition. For the WLF fits, $T_{s}=142{ }^{\circ} \mathrm{C}$. Error bars represent uncertainty in temperature $\left( \pm 0.3{ }^{\circ} \mathrm{C}\right)$ and in the temperature shift factor based on the expanded uncertainty for repeat measurements at a single temperature.

Clearly, the data from the present study show a significantly smaller temperature dependence than either of the two WLF curves. Further, the data are almost linear with temperature. We also fitted the data to a WLF function with $T_{s}$ $=142^{\circ} \mathrm{C}$ using a least squares fitting procedure to give values of $C_{1}=49.7$ and $C_{2}=174.6^{\circ} \mathrm{C}$. The resulting curve is also plotted in Fig. 5. In terms of the Vogel-Fulcher temperature singularity $T_{\infty}$, we find that the value is far below that normally expected for glass forming liquids relative to the glass transition temperature, $T_{\infty}=T_{s}-C_{2} \approx-33^{\circ} \mathrm{C}=T_{g}$ $-175^{\circ} \mathrm{C}$. In Fig. 6 we replot the data on an Arrhenius plot $\left(\log a_{T} \mathrm{vs} 1 / T\right)$ and show the WLF behavior calculated from the Mercier and Groenincks ${ }^{45}$ parameters as well as a line representing the Arrhenius fit to the data with resulting activation energy of $E_{a}=9.58 \times 10^{5} \mathrm{~J} \mathrm{~mol}^{-1} \mathrm{~K}^{-1}$. Although the data cannot be used to distinguish among a linear temperature dependence, an Arrhenius temperature dependence or a WLF-Vogel-Fulcher temperature dependence with a very low singularity temperature, it is clear that the equilibrium temperature dependence of the segmental viscoelastic relaxation of polycarbonate does not follow the expected strong singularity obtained from measurements above the glass transition. We interpret this result to be consistent with the predictions of the DiMarzio-Yang ${ }^{9}$ model. (See also the discussion in Ref. 33.)

\section{DISCUSSION}

The strongly non-Arrhenius temperature dependence of the viscosity or relaxation times has long been assumed to be the hallmark of glass forming liquids. Therefore, it is of interest to ask what the significance of the current results is. First, of course, the question arises as to the universality of the observed transition from the WLF-Vogel-Fulcher-type 


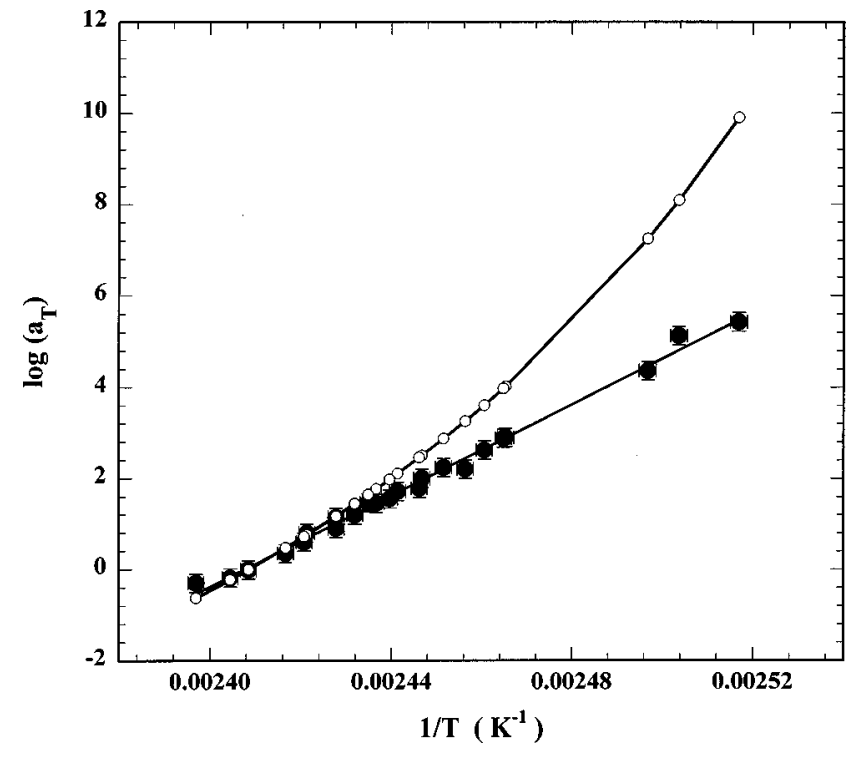

FIG. 6. Arrhenius plot of the data given in Fig. 5. Solid points are data, solid line is Arrhenius fit to the data. Line with open circles is a WLF equation obtained by Mercier and Gronincks (Ref. 45) for the segmental relaxation of bisphenol A polycarbonate.

behavior to the Arrhenius behavior. Is it unique to, e.g., polycarbonate? Or are there other instances of such observed behavior? Do other measurements that observe Arrhenius behavior extend to the same temperature regime? And so forth.

There are sporadic reports of some glass forming liquids exhibiting Arrhenius-temperature dependencies near to the glass transition. Macedo and Napolitano ${ }^{23}$ have reported that $\mathrm{B}_{2} \mathrm{O}_{3}$ glasses exhibit a transition in viscosity to Arrhenius behavior below the glass transition temperature. However, unlike the polycarbonate studied here, $\mathrm{B}_{2} \mathrm{O}_{3}$ exhibits other unusual thermodynamic behavior as well. For example the entropy of $\mathrm{B}_{2} \mathrm{O}_{3}$ does not extrapolate to a lower entropy than the crystal form of the material at the so-called Kauzmann ${ }^{46}$ temperature. Furthermore, the volume-temperature response of $\mathrm{B}_{2} \mathrm{O}_{3}$ does not exhibit the normal behavior ${ }^{47}$ above the glass transition, i.e., it is concave towards the temperature axis in a plot of volume vs temperature rather than convex as in polycarbonate and other glass forming polymers. ${ }^{5,29}$

In other work, Laughlin and Uhlmann ${ }^{24}$ and Cukierman et al $^{48}$ have reported for a series of organic glass forming liquids that the viscosity-temperature dependence follows Arrhenius-type behavior beginning at temperatures well above the nominal glass transition temperature. For example, Arrhenius-type behavior is exhibited by $\alpha$-phenyl-o-cresol at $\eta>10^{4} \mathrm{Pas}$ which corresponds to a temperature approximately $20^{\circ} \mathrm{C}$ above the glass transition measured calorimetrically and approximately $35^{\circ} \mathrm{C}$ above the glass transition temperature (taken as the point at which the viscosity is $10^{12} \mathrm{~Pa} \mathrm{~s}$, a common practice ${ }^{2,23,24,46}$. In the same series of studies, large deviations from the Vogel-Fulcher-type temperature dependence of the viscosity were also shown for ortho-terphenyl, salol and tri- $\alpha$-naphthylbenzene. However, in all instances, the onset appeared well above the glass transition temperature and the $T_{\infty}$ values were reported to be very close to the glass transition temperature whether based on calorimetric estimates or on the point at which the viscos-
TABLE II. Comparison of the calorimetric (or volumetric) glass transition temperature, the viscosity ${ }^{\mathrm{a}}$ (glass transition), and the Vogel-Fulcher singularity temperature for various glass-forming materials. References given in parentheses.

\begin{tabular}{|c|c|c|c|}
\hline Material & $\begin{array}{c}T_{g} / \mathrm{K} \\
\text { (Calorimetric) }\end{array}$ & $\begin{array}{c}T_{g} / \mathrm{K} \\
\left(\eta=10^{12} \mathrm{Pas}\right)\end{array}$ & $T_{\infty} / \mathrm{K}$ \\
\hline $\begin{array}{l}\text { Bisphenol A } \\
\text { Polycarbonate }\end{array}$ & $414.5(36)$ & N/A & $371.2(45)$ \\
\hline Polystyrene & $370(1)$ & N/A & $325(1)$ \\
\hline Polybutadiene & $261(1)$ & N/A & $226(1)$ \\
\hline Poly(vinyl acetate) & $305(1)$ & N/A & $258(1)$ \\
\hline $\begin{array}{l}\text { Poly(methyl } \\
\text { methacrylate) }\end{array}$ & $388(1)$ & N/A & $308(1)$ \\
\hline Ortho-terphenyl & $248(48)$ & $241(24,48)$ & $\begin{array}{ll}248 & (24,48) \\
231 & (49)\end{array}$ \\
\hline Salol & $220(24)$ & 213 & $226(24)$ \\
\hline$\alpha$-phenyl-o-cresol & $220(24)$ & $210(48)$ & $210(24)$ \\
\hline $\begin{array}{l}\text { Tri- } \alpha \text {-naphthyl- } \\
\text { benzene }\end{array}$ & $\cdots$ & $334(48)$ & $342.7(24)$ \\
\hline $\mathrm{B}_{2} \mathrm{O}_{3}$ & 555 & $548(23)$ & $138(23)$ \\
\hline $\begin{array}{l}1,2 \text { diphenyl } \\
\text { benzene }\end{array}$ & $\begin{array}{l}240.8(51) \\
\text { [volumetric] }\end{array}$ & $\cdots$ & $171.9(51)$ \\
\hline 6-phenyl ether & $\begin{array}{l}248.2(51) \\
\text { [volumetric] }\end{array}$ & $\cdots$ & $173.4(51)$ \\
\hline $\begin{array}{l}\text { Tri-cresyl } \\
\text { phosphate }\end{array}$ & $\begin{array}{l}156.5(51) \\
\text { [volumetric] }\end{array}$ & $\cdots$ & $200.2(51)$ \\
\hline
\end{tabular}

a Defined as temperature at which $\eta=10^{12} \mathrm{~Pa}$ s. Also, note that this measure is not applicable to polymers because their viscosity reflects the entanglement mechanism and varies dramatically with molecular weight while other measures of the glass transition are relatively insensitive to molecular weight once the system is well entangled (Ref. 1).

ity reached a value of $10^{12} \mathrm{~Pa}$ s. Table II shows ${ }^{1,17,23,24,49,50,51}$ some comparisons of the calorimetric and viscosity estimated $T_{g}$ values as well as the Vogel-Fulcher $T_{\infty}$ for several glass forming materials. As noted previously, $T_{\infty}$ is often in the vicinity of $50{ }^{\circ} \mathrm{C}$ below the $T_{g}$ as is the case for the polycarbonate data obtained by Mercier and Groenincks. ${ }^{45}$ By comparison, the polycarbonate data obtained in this study suggest strongly that the segmental relaxation changes its temperature dependence from WLF-Vogel-type to Arrhenius-type at the same point as the structural recovery times become such that the polymer undergoes its kinetic glass transition.

Also, there are other recent measurements on polymers leading to similar findings to our own. Simon et al. ${ }^{25}$ report on structural recovery data for polystyrene and find a similar trend to that observed for our polycarbonate data. In addition, they report that data from Braun and Kovacs, ${ }^{52}$ upon reanalysis of the temperature dependence of the shift factors, show a transition from Vogel-Fulcher to Arrhenius-type behavior in the vicinity of the conventionally measured glass transition. We remark that Braun and Kovacs ${ }^{52}$ did not come to the same conclusion.

In addition, we note that a recent paper by Stickel et al. ${ }^{53}$ reports the results of an investigation of the temperature dependence of simple liquid dynamics using multiple methods of measurement. They concluded that there is a transition from the Vogel-Fulcher behavior to a second VogelFulcher behavior when the glass transition does not intervene. Our results for a polymeric glass suggest that if there is 
a second Vogel-Fulcher regime, it must appear well below the glass transition or have a temperature dependence closely approximating the Arrhenius-type of temperature dependence.

Finally, we remark that the polymer dynamics model of Douglas and Hubbard ${ }^{54}$ suggests a return to Arrhenius behavior below $T_{g}$ followed by a second non-Arrhenius regime below $T_{\infty}$-a regime inaccessible in this study. The reader is referred to that work for further details.

\section{SUMMARY}

Investigation of the time-temperature superposition response of a polycarbonate glass below the glass transition shows that the temperature dependence of the equilibrium shift factors deviates strongly from the singular behavior expected from the Vogel-Fulcher or WLF dependence obtained in equilibrium and above the nominal $T_{g}$. Samples were aged into equilibrium at temperatures as much as $17^{\circ} \mathrm{C}$ below the nominal calorimetric $T_{g}$ and the segmental shift factors were obtained from the stress relaxation response. The temperature dependence of the shift factors in the temperature range investigated can be interpreted as being Arrhenius, but is also indistinguishable from either a linear temperature dependence or a very weak singularity, with Vogel-Fulcher parameter $T_{\infty}$ having a value some $175^{\circ} \mathrm{C}$ below the nominal $T_{g}$. These results are consistent with a new model developed by DiMarzio and Yang ${ }^{9}$ in which it is claimed that the viscosity should not exhibit a temperature singularity in traversing the glass transition.

${ }^{1}$ J. D. Ferry, Viscoelastic Properties of Polymers, 3rd ed. (Wiley, New York, 1980).

${ }^{2}$ C. A. Angell, J. Res. Natl. Inst. Stand. Technol. 102, 171 (1997).

${ }^{3}$ H. Vogel, Phys. Z. 22, 645 (1921); G. S. Fulcher, J. Am. Ceram. Soc. 8, 339 (1925).

${ }^{4}$ M. L. Williams, R. F. Landel, and J. D. Ferry, J. Am. Chem. Soc. 77, 3701 (1955).

${ }^{5}$ G. B. McKenna, "Glass formation and glassy behavior," in Comprehensive Polymer Science, edited by C. Booth and C. Price (Pergamon, Oxford, 1989), Vol. 2, p. 311.

${ }^{6}$ G. B. McKenna, G. Hadziioannou, P. Lutz, G. Hild, C. Strazielle, C. Straupe, P. Rempp, and A. J. Kovacs, Macromolecules 20, 498 (1987).

${ }^{7}$ A. K. Doolittle, J. Appl. Phys. 22, 1471 (1951).

${ }^{8}$ G. Adam and J. H. Gibbs, J. Chem. Phys. 43, 139 (1965).

${ }^{9}$ E. A. DiMarzio and A. J. M. Yang, J. Res. Natl. Inst. Stand. Technol. 102, 135 (1997).

${ }^{10}$ D. J. Plazek, X. D. Zheng, and K. L. Ngai, Macromolecules 25, 4920 (1992).

${ }^{11}$ P. G. Santangelo and C. M. Roland, Macromolecules 31, 3715 (1998).

${ }^{12}$ R. Zorn, G. B. McKenna, L. Willner, and D. Richter, Macromolecules 28 , 8552 (1995)

${ }^{13}$ K. L. Ngai and D. J. Plazek, Rubber Chem. Technol. 68, 376 (1995).

${ }^{14}$ K. L. Ngai, D. J. Plazek, and R. W. Rendell, Rheol. Acta 36, 307 (1997).

${ }^{15}$ A. Lee and G. B. McKenna, Polymer 29, 1812 (1988).

${ }^{16} \mathrm{G}$. W. Scherer, Relaxation in Glass and Composites (Wiley, New York, 1986).

${ }^{17}$ C. T. Moynihan, J. Am. Ceram. Soc. 76, 1081 (1993).

${ }^{18}$ P. A. O'Connell and G. B. McKenna, Polym. Eng. Sci. 37, 1485 (1997).

${ }^{19}$ H. G. Merriman and J. M. Caruthers, J. Polym. Sci., Polym. Phys. Ed. 19, 1055 (1981).

${ }^{20}$ P. A. O'Connell and G. B. McKenna, “Time-Temperature Superposition at Equilibrium in Polycarbonate," in Proceedings of the NATAS 25th Annual Conference, September, 1997 (Omnipress, Madison, WI, 1997), pp. $420-427$.
${ }^{21}$ P. A. O'Connell and G. B. McKenna, Soc. Plastics Eng. ANTEC 98 II, 2152 (1998)

${ }^{22}$ L. C. E. Struik, Physical Aging in Polymers and Other Amorphous Materials (Elsevier, Amsterdam, 1976).

${ }^{23}$ P. B. Macedo and A. Napolitano, J. Chem. Phys. 49, 1887 (1968).

${ }^{24}$ W. T. Laughlin and D. R. Uhlmann, J. Phys. Chem. 76, 2317 (1972).

${ }^{25}$ S. L. Simon, J. W. Sobieski, and D. J. Plazek (private communication).

${ }^{26}$ R. Kohlrausch, Ann. Phys. Chem. J. C. Poggendorff 91, 179 (1854).

${ }^{27}$ G. Williams and D. C. Watts, Trans. Faraday Soc. 66, 80 (1970).

${ }^{28}$ We remark that while time-temperature superposition for this polycarbonate seems valid, the KWW function [Eq. (2)] was not an adequate descriptor for the master curve. The results of the prior study showed isothermal adherence to a constant $\beta$ and, hence, time-aging time superposition and KWW relaxation behavior. This observation does not invalidate the time-temperature superposition, it does indicate that the KWW function is only an approximate descriptor of the material responses. The reader is referred to Ref. 18 for further details.

${ }^{29}$ D. J. Plazek and A. J. Chelko, Polymer 18, 15 (1977).

${ }^{30}$ H. Markovitz, J. Polym. Sci., Polym. Symp. 50, 431 (1975).

${ }^{31}$ J. H. Gibbs and E. A. DiMarzio, J. Chem. Phys. 28, 373 (1958).

${ }^{32}$ E. A. DiMarzio and J. H. Gibbs, J. Chem. Phys. 28, 807 (1958).

${ }^{33}$ Dr. J. F. Douglas of the NIST suggested to us that, in fact, the AdamGibbs relations can give an Arrhenius-type temperature dependence below $T_{g}$. If one assumes, as found in recent computer simulations (Ref. 38) that the configurational entropy does not vanish at $T_{g}$ but becomes constant. Then the viscosity below $T_{g}$ would look like $\eta \sim \exp \left[A / S_{c} T\right]$ or $a_{T}$ $\sim \exp \left[\left(A / S_{c}\right)\left(1 / T-1 / T_{g}\right)\right]$ which is of the same form as Eq. (12).

${ }^{34}$ J. Baschnagel, M. Wolfgardt, W. Paul, and K. Binder, J. Res. Natl. Inst. Stand. Technol. 102, 149 (1997).

${ }^{35}$ Certain commercial materials and equipment are identified in this paper in order to specify adequately the experimental procedure. In no case does such identification imply recommendation or endorsement by the National Institute of Standards and Technology, nor does it imply necessarily the best for the purpose.

${ }^{36}$ J.-J. Pesce, J. M. Niemiec, M. Y. Chiang, C. L. Schutte, C. R. Schultheisz, and G. B. McKenna, "Characterization of Polymers in the Glass Transition Range: Time-Temperature and Time-Aging Time Superposition in Polycarbonate," in Current Research in the Thermo-Mechanics of Polymers in the Rubbery-Glassy Range, edited by M. Negahban (American Society of Mechanical Engineers, New York, 1995), AMD-Vol. 203, p. 77.

${ }^{37}$ D. M. Colucci, P. A. O'Connell, and G. B. McKenna, Polym. Eng. Sci. 37, 1469 (1997).

${ }^{38}$ D. M. Colucci, G. B. McKenna, J. J. Filliben, A. Lee, D. B. Curliss, K. B. Bowman, and J. D. Russell, J. Polym. Sci., Polym. Phys. Ed. 35, 1561 (1997).

${ }^{39}$ G. B. McKenna and A. J. Kovacs, Polym. Eng. Sci. 24, 1131 (1984).

${ }^{40}$ D. J. Plazek, J. Phys. Chem. 69, 3480 (1965).

${ }^{41}$ P. A. O'Connell, C. R. Schultheisz, and G. B. McKenna, "The Physics of Glassy Polycarbonate: Superposability and Volume Recovery," in The Physics of Glassy Polymers, edited by M. Tant and A. Hill (American Chemical Society, Washington, D.C., 1999), pp. 199-217.

${ }^{42}$ A. Lee and G. B. McKenna, Polymer 31, 423 (1990).

${ }^{43}$ L. C. E. Struik, Polymer 29, 1347 (1998).

${ }^{44}$ I. Echeverria, P. C. Su, S. L. Simon, and D. J. Plazek, J. Polym. Sci., Part B: Polym. Phys. 33, 2457 (1995).

${ }^{45}$ J. Mercier and G. Groenincks, Rheol. Acta 8, 511 (1969).

${ }^{46}$ W. Kauzmann, Chem. Rev. 43, 219 (1948).

${ }^{47}$ P. B. Macedo, W. Capps, and T. A. Litovitz, J. Chem. Phys. 44, 3357 (1966).

${ }^{48}$ M. Cukierman, J. W. Lane, and D. R. Uhlmann, J. Chem. Phys. 59, 3639 (1973).

${ }^{49}$ R. J. Greet and D. Turnbull, J. Chem. Phys. 46, 1243 (1967).

${ }^{50}$ C. L. Jackson and G. B. McKenna, J. Non-Cryst. Solids 131, 221 (1991).

${ }^{51}$ D. J. Plazek, C. A. Bero, and I.-C. Chay, J. Non-Cryst. Solids 171-174, 181 (1994).

${ }^{52}$ G. Braun and A. J. Kovacs, Phys. Chem. Glasses 4, 152 (1963).

${ }^{53}$ F. Stickel, E. W. Fischer, and R. Richert, J. Chem. Phys. 104, 2043 (1996)

${ }^{54}$ J. F. Douglas and J. B. Hubbard, Macromolecules 24, 3163 (1991). 